\title{
Pharmacokinetics, Pharmacodynamics, and Immunogenicity of Belatacept in Adult Kidney Transplant Recipients
}

\author{
Jinshan Shen - Robert Townsend $\cdot$ Xiaoli You $\cdot$ Yun Shen $\cdot$ Ping Zhan • \\ Zexun Zhou • Dong Geng • Dianna Wu • Nadia McGirr • Kathleen Soucek • \\ Elizabeth Proszynski $\cdot$ Janice Pursley $\cdot$ Eric Masson
}

Published online: 12 November 2013

(C) The Author(s) 2013. This article is published with open access at Springerlink.com

\begin{abstract}
Background and Objectives Belatacept is a first-in-class, selective co-stimulation blocker recently approved for the prophylaxis of organ rejection in adult kidney transplant recipients. The objective of this study was to report the pharmacokinetics, pharmacodynamics, and immunogenicity of belatacept.

Methods The pharmacokinetics, pharmacodynamics (CD86 receptor occupancy), and immunogenicity of belatacept were studied in de novo adult kidney transplant recipients in phase II and III clinical studies.

Results Following multiple doses of 5 or $10 \mathrm{mg} / \mathrm{kg}$, the geometric mean (percentage coefficient of variation) maximum serum concentration and area under the serum concentration-time curve over one dosing interval of belatacept were $136(20 \%)$ and $238(27 \%) \mu \mathrm{g} / \mathrm{mL}$, and $13,587(27 \%)$ and $21,241(35 \%) \mu \mathrm{g} \cdot \mathrm{h} / \mathrm{mL}$, respectively. The median belatacept elimination half-life was 8-9 days. Belatacept exhibited concentration-dependent binding to CD86 receptors. The pre-dose CD86 receptor occupancy by belatacept decreased from 94 to $65 \%$ between day 5 and 1 year post-transplant, with corresponding pre-dose trough serum concentrations of belatacept decreasing from $\sim 35$ to $4 \mu \mathrm{g} / \mathrm{mL}$ during this period. The cumulative incidence of developing anti-belatacept antibodies was $5.3 \%$ up to 3 years post-transplant and had no impact on belatacept exposure.
\end{abstract}

J. Shen $\cdot$ R. Townsend $\cdot$ X. You - Y. Shen $\cdot$ P. Zhan · Z. Zhou ·

D. Geng · D. Wu · N. McGirr - K. Soucek · E. Proszynski ·

J. Pursley $\cdot$ E. Masson $(\varangle)$

Clinical Pharmacology and Pharmacometrics,

Oncology-Immunology, Bristol-Myers Squibb,

P.O. Box 4000, E1.409, Princeton, NJ 08543-4000, USA

e-mail: eric.masson@bms.com
Conclusions Belatacept in adult kidney transplant demonstrated linear pharmacokinetics with low variability, concentration-dependent pharmacodynamics, and a low incidence of anti-drug antibodies.

\section{Introduction}

Although advances in post-transplant immunosuppression have reduced the rates of acute rejection and improved 1 -year outcomes, commensurate improvements in longterm renal allograft survival rates have not been observed [1]. Calcineurin inhibitor (CNI)-based immunosuppression in kidney transplant recipients (KTRs) is associated with toxicities such as nephrotoxicity, hypertension, dyslipidemia, and diabetes mellitus, which limit long-term outcomes [2]. In addition, therapeutic drug monitoring (TDM) of CNIs is required because of their narrow therapeutic index, significant risk of drug-drug interactions, and high exposure variability after oral dosing, all of which add to the overall burden for the patient [3]. Consequently, there is a significant need for new immunosuppressive therapies to provide effective long-term immunosuppression with reduced nephrotoxicities and pharmacokinetic and pharmacodynamic characteristics that do not require TDM [2].

Belatacept (LEA29Y, NULOJIX ${ }^{\circledR}$, Bristol-Myers Squibb, Princeton, NJ, USA), a fusion protein combining a modified cytotoxic T-lymphocyte-associated antigen 4 (CTLA-4) extracellular domain with the constant-region fragment of human immunoglobulin G1, is a first-in-class, selective co-stimulation blocker recently approved for the prophylaxis of organ rejection in adult KTRs [4]. Belatacept binds to CD80 and CD86 receptors on the antigenpresenting cell (APC) surface with high specificity and affinity, thereby blocking the interaction between CD80/ 
CD86 and CD28 on T cells [5]. In doing so, belatacept prevents $\mathrm{T}$ cell activation and proliferation and inhibits subsequent alloimmune responses following organ transplantation [6]. The interaction of a drug with its biologic target (e.g., receptor saturation) has been previously used as a pharmacodynamic biomarker of target engagement in drug development [7]. In vitro studies demonstrated that inhibition of alloimmune responses by belatacept was more closely correlated with its CD86 receptor occupancy than CD80 receptor occupancy, suggesting that CD86 receptor occupancy may be a useful surrogate marker for inhibition of alloimmune responses by belatacept and thus serve as a measure of pharmacodynamic activity in KTRs [6].

The characterization of the pharmacokinetics, pharmacodynamics, and immunogenicity of belatacept provides insights into the exposure-response relationship of efficacy and safety of belatacept and the mechanism of action in vivo, and supports appropriate clinical dosing in KTRs. Here we report the pharmacokinetics, pharmacodynamics, and immunogenicity of belatacept in de novo KTRs from several phase II and III clinical studies.

\section{Methods}

\subsection{Studies Included in Analyses}

Data from several belatacept clinical studies are reported here: a phase II open-label pharmacokinetic study $(N=12$ subjects treated and ten completing week 16 visit procedures) [8]; a phase II randomized corticosteroid-avoidance study $(N=62)$ [9]; the pharmacokinetic substudy of a phase II multiple-dose efficacy and safety study $(N=14)$ [10]; the 3-year randomized, partially blinded phase III
BENEFIT (Belatacept Evaluation of Nephroprotection and Efficacy as First-line Immunosuppression Trial) [11, 12] and BENEFIT-EXT (Belatacept Evaluation of Nephroprotection and Efficacy as First-Line Immunosuppression Trial-Extended Criteria Donors) [12, 13] studies assessing a less intensive (LI) and a more intensive (MI) dosing regimens of belatacept versus ciclosporin in adults receiving a kidney transplant $(N=804)$; and the long-term extension (LTE) of a phase II dose-finding study $(N=102)$ [14]. All patients were de novo KTRs receiving kidneys from living donors, standard criteria donors (deceased donors with an anticipated cold ischemia time of $<24 \mathrm{~h}$ ), or extended criteria donors [donors $\geq 60$ years old, or donors $\geq 50$ years old and who had at least two other risk factors (cerebrovascular accident, hypertension, or serum creatinine $>1.5 \mathrm{mg} / \mathrm{dL}$ ); or an anticipated cold ischemia time of $\geq 24 \mathrm{~h}$ or donation after cardiac death]. Belatacept was administered in all studies without TDM, while ciclosporin was administered with TDM [15]. The studies were approved by institutional review boards, and all patients provided signed informed consent, per institutional guidelines.

\subsection{Belatacept Dosing Regimens}

Two belatacept dosing regimens, LI and MI, were tested in the phase III program. Both the LI and MI dosing regimens involved belatacept administration via a 30-min intravenous infusion at a dose of $10 \mathrm{mg} / \mathrm{kg}$ during the initial phase and $5 \mathrm{mg} / \mathrm{kg}$ during the maintenance phase post-transplant. However, in contrast to the MI regimen, the approved belatacept LI regimen had less frequent dosing during the initial phase, and the maintenance phase started earlier (Fig. 1).
Fig. 1 Belatacept MI and LI dosing regimens in the phase III BENEFIT and BENEFIT-EXT studies. Each solid vertical line up to days 168 (MI) and 84 (LI) post-transplant represents an intravenous infusion dose of belatacept $10 \mathrm{mg} / \mathrm{kg}$. Starting from days 197 (MI) and 112 (LI), a maintenance intravenous infusion dose of belatacept $5 \mathrm{mg} / \mathrm{kg}$ was administered every 4 weeks. An infusion dose of placebo was administered on days 42 and 70 of the LI regimen to maintain the blinding of the LI and MI regimens in both studies. $L I$ less intensive, $M I$ more intensive
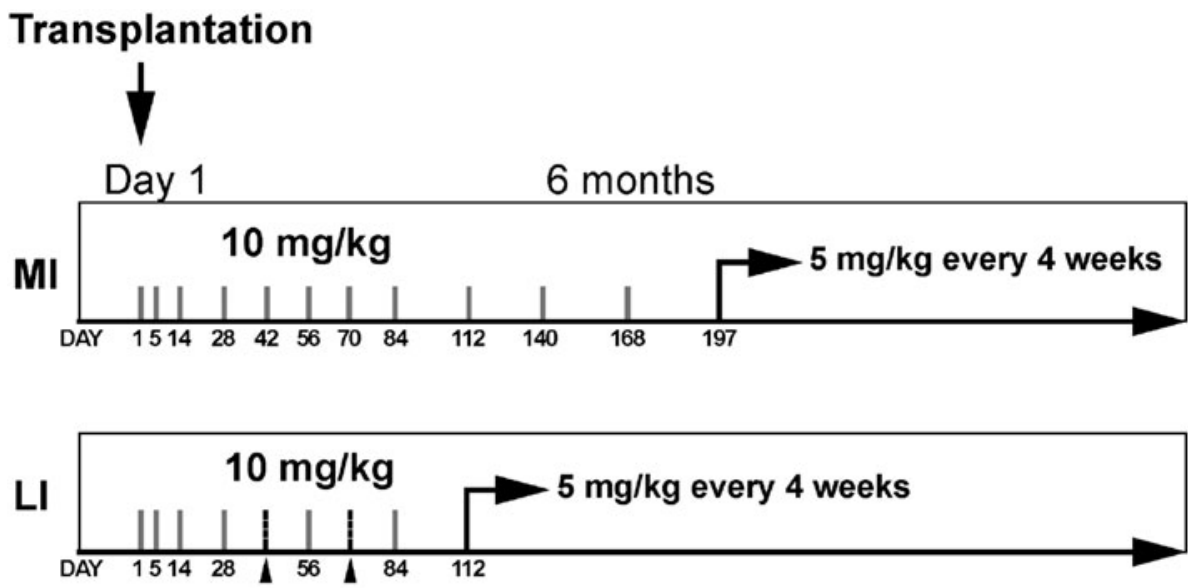

$\Lambda$ Placebo infusions 


\subsection{Pharmacokinetic Bioanalytical Methods}

Details of the enzyme-linked immunosorbent assay (ELISA) for belatacept quantification in human serum samples were reported previously [16]. Blood samples (3-5 mL) for pharmacokinetic assessments were collected from an indwelling catheter or by direct venipuncture and processed for serum. Total serum belatacept concentrations were determined using a validated ELISA method. The lower limit of quantification (LLOQ) and upper limit of quantification were established at 3.0 and $80.0 \mathrm{ng} / \mathrm{mL}$, respectively. Between-run and within-run percentage coefficient of variation (CV\%) were $\leq 11.81$ and $\leq 20.78 \%$, respectively. All belatacept serum samples were shipped and analyzed at PPD (Richmond, VA, USA).

\subsection{Pharmacokinetic Analyses}

Pharmacokinetic analyses were performed on belatacept concentration versus time data in KTRs following multiple 5 or $10 \mathrm{mg} / \mathrm{kg}$ intravenous belatacept infusions.

Pharmacokinetic analysis for the $5 \mathrm{mg} / \mathrm{kg}$ dose was performed on data from the pharmacokinetic substudy of the phase II LTE study; blood samples were collected from 14 patients following the administration of the first dose in the pharmacokinetic substudy (day 1 of the substudy) at the following timepoints: pre-dose $(0 \mathrm{~h})$, end of infusion $(0.5 \mathrm{~h}), 2$ and $8 \mathrm{~h}$, and 1, 3, 6, 13, 20, and 27 days post-dose.

Pharmacokinetic analysis for the $10 \mathrm{mg} / \mathrm{kg}$ dose was performed on data from an open-label pharmacokinetic study in ten de novo KTRs; blood samples were collected between the week 12 dose and the week 16 dose at pre-dose, at end of infusion (0.5), at $2 \mathrm{~h}$, and at 3, 7, 14, 21, and 28 days following the week 12 dose. Pharmacokinetic sampling was also performed to determine belatacept trough serum concentrations $\left(C_{\text {trough }}\right)$ in the phase III studies BENEFIT and BENEFIT-EXT, for which blood samples were collected pre-dose on days 1 and 5 and at weeks $8,12,16,24,36,52$, 104, 128, and 156. Dosing windows of belatacept were prospectively specified in both phase III studies to provide KTRs flexibility on intravenous infusion time. The dosing windows allowed were $\pm 6 \mathrm{~h}, \pm 2$ days, \pm 3 days, and \pm 5 days for day 5 , week 2 , week 4 to month 6 , and months 6 to 36 post-transplant, respectively.

Individual subject steady-state pharmacokinetic parameters were derived using non-compartmental methods by the validated pharmacokinetic analysis program Kinetica ${ }^{\circledR}$ (Thermo Fisher Scientific Inc., Waltham, MA, USA). Actual sampling times were used for pharmacokinetic calculations, and nominal times were used for generation of mean serum concentration-time plots and summaries. For the purpose of calculating pharmacokinetic parameters, predose concentrations below the LLOQ and concentrations prior to the first quantifiable concentration that were below the LLOQ were set to "zero." All other concentrations below the LLOQ were set to "missing" for purposes of tabular presentation and graphing mean profiles. The LLOQ value for belatacept was $3 \mathrm{ng} / \mathrm{mL}$. The pharmacokinetic parameters maximum serum concentration $\left(C_{\max }\right)$ and $C_{\text {trough }}$ were recorded directly from experimental observations. Using no weighting factor, the terminal log-linear phase of the concentration-time curve was identified by least-square linear regression of at least three datapoints that yielded the maximum G-criteria, which is also referred to as "adjusted R-squared." The serum elimination half-life $\left(t_{1 / 2}\right)$ was calculated as $\ln 2 / \lambda_{z}$, where " $\lambda_{z}$ " was the terminal elimination rate constant. The area under the serum concentration-time curve over one dosing interval $\left(\mathrm{AUC}_{\tau}\right)$ was calculated by combining log- and linear-trapezoidal summations. Total body clearance (CL), was calculated by dividing the dose by $\mathrm{AUC}_{\tau}$. Volume of distribution at steady state $\left(V_{\mathrm{ss}}\right)$ was calculated by dividing the dose by $\mathrm{AUC}_{\tau}$ and multiplying by the mean residence time.

For statistical analysis of $C_{\text {trough }}$, all concentrations less than LLOQ were imputed to LLOQ/2. Geometric mean and $\mathrm{CV} \%$ were reported for $C_{\max }, \mathrm{AUC}_{\tau}, \mathrm{CL}, \mathrm{V}_{\mathrm{ss}}$, and $C_{\text {trough }}$. Median and range were reported for $t_{1 / 2}$.

\subsection{Pharmacodynamic Analyses}

The CD86 receptor occupancy by belatacept on whole peripheral blood monocytes was evaluated in a phase II corticosteroid-avoidance study in de novo KTRs receiving the MI regimen [9]. Whole blood samples were obtained prior to dosing at baseline, day 5 , and weeks $2,4,12,24$, and 52 posttransplant (all pre-infusion). Approximately $8.5 \mathrm{~mL}$ of whole blood were collected into acid citrate dextrose Vacutainer ${ }^{\circledR}$ [Becton Dickinson \& Co. (BD), Franklin Lakes, NJ, USA] tubes and analyzed within $48 \mathrm{~h}$ of collection.

\subsection{CD86 Receptor Occupancy Assay}

CD86 receptor occupancy by belatacept was assessed via modification of a previously established whole blood flow cytometric-based CD86 receptor competition assay using phycoerythrin (PE)-conjugated antihuman CD86 monoclonal antibody clone HA5.2B7 (HA5-PE) [6]. Samples were processed in duplicate and evaluated on a FACSCanto $^{\mathrm{TM}}$ flow cytometer using DIVA ${ }^{\mathrm{TM}}$ analysis software (BD, San Jose, CA, USA). Data were analyzed with FlowJo $^{\text {TM }}$ analysis software (Tree Star, Ashland, OR, USA). Mean fluorescence intensity of free CD86 on CD14+ monocytes was converted to molecules of equivalent soluble fluorochrome (MESF). The free CD86 level on monocytes was computed by subtracting the HA5 background fluorescence from the total HA5 fluorescence 
( $\Delta$ HA5-PE) for each time point and for each KTR in the study.

The percentage of CD86 receptor occupancy at each timepoint was computed using the free CD86 receptor level at baseline and at each timepoint post-transplant with Eq. 1:

$\% \mathrm{CD} 86$ receptor occupancy $=\left[1-\left(\frac{\Delta \mathrm{HA} 5_{\text {posttransplant }}}{\Delta \mathrm{HA} 5_{\text {baseline }}}\right)\right]$

$$
\times 100
$$

An inhibitory maximum effect $\left(E_{\max }\right)$ model was used to describe the free CD86 receptor-belatacept concentration relationship (Eq. 2):

$R(C)=E_{0}-\frac{E_{\max } \times C}{E C_{50}+C}$

where response $R(C)$ was the free CD86 receptor level at a belatacept concentration of $C, E_{0}$ was the baseline free CD86 receptor level when drug concentration is $0, E_{\max }$ was the maximal decrease in free CD86 receptor level, $E C_{50}$ was the belatacept concentration required to achieve $50 \%$ of $E_{\max }$, and $C$ was belatacept concentration. $E C_{90}$ was the belatacept concentration required to achieve $90 \%$ of $E_{\max }$. The maximal CD86 receptor occupancy by belatacept was calculated as $E_{\max } / E_{0} \times 100 \%$.

This inhibitory $E_{\max }$ model was used to account for intersubject variability with random effect and implemented with a non-linear mixed effect approach on $\mathrm{S}+$ statistical analysis software (TIBCO Spotfire, Somerville, MA, USA). To account for potential heteroscedastic residual error variance, exponential variance function structure was employed. Effect of within-subject correlation was considered using exponential spatial correlation. The $95 \%$ confidence intervals (CIs) of parameter estimates were calculated using both normal approximation and bootstrap methods to confirm estimation adequacy. The 2.5th to 97.5th percentile CI around the fitted mean was constructed using bootstrap. The impact of random effect, error variance covariate function, and correlation structure was also evaluated. The final model was selected based on the log-likelihood criterion using the differences in $-2 \times \log$ of likelihood between an original and alternative model given the difference was asymptotically chi-squared $\left(\chi^{2}\right)$ distributed.

\subsection{Immunogenicity Assessment}

Immunogenicity data were assessed throughout the initial and maintenance phase in 796 KTRs in two phase III studies (BENEFIT and BENEFIT-EXT) [11, 13] with a median of 3.3 years of exposure to belatacept and in 51 KTRs in the phase II LTE study with exposure to belatacept for approximately 7 years [14].
Samples for analysis of anti-belatacept antibodies were collected pre-treatment and throughout treatment, simultaneously with the collection of sera samples to determine belatacept $C_{\text {trough }}$ (months 3 and 6 post-transplant, and every 6 months thereafter), and at the 8-week follow-up visit for KTRs who discontinued belatacept treatment.

The potential impact of anti-belatacept antibodies on belatacept pharmacokinetics in KTRs was assessed in the phase II LTE and the phase III BENEFIT and BENEFIT-EXT studies. Individual Bayesian-predicted clearance of belatacept from population pharmacokinetic analyses [17] was determined and assessed graphically using a box and whisker plot for KTRs with the following anti-belatacept antibody status: seropositive, seronegative, indeterminate (neither positive nor negative), neutralizing antibodies, and antibodies specific to the modified CTLA-4 portion of belatacept.

\subsection{Immunogenicity Assay}

A validated electrochemiluminescence bridging assay was employed to detect anti-belatacept antibodies from sera collected at $C_{\text {trough }}$ just prior to the next dose. All potentially positive samples detected in a screening assay were tested in a confirmatory immunodepletion assay with belatacept, LEA29Y-T, and CD40Ig to exclude false-positives. Samples confirmed to be positive were titrated to determine the titer of anti-belatacept antibodies. The assay had a sensitivity of $12.5 \mathrm{ng} / \mathrm{mL}$ in belatacept-free sera and could detect $250 \mathrm{ng} / \mathrm{mL}$ of anti-belatacept antibodies in the presence of up to $10 \mu \mathrm{g} / \mathrm{mL}$ of belatacept, which allowed detection of anti-belatacept antibodies in the majority of subjects by day 56 post-transplant as mean belatacept $C_{\text {trough }}$ values were below that cutoff by that time for both the MI and LI regimens. Seropositive samples with reactivity to the modified CTLA-4 portion of belatacept were further characterized using validated bioassays to determine whether the anti-belatacept antibodies could neutralize belatacept activity (i.e., neutralizing antibodies). This was assessed in a co-stimulation-dependent bioassay that was dependent on the interaction of CD28 and CD80/ CD86 and compared the response of a seropositive serum sample with its corresponding pre-treatment sample. All samples for anti-belatacept antibodies were shipped and analyzed at Tandem Labs (West Trenton, NJ, USA).

\section{Results}

\subsection{Pharmacokinetics of Belatacept in Kidney Transplant Recipients (KTRs)}

In the phase III clinical studies BENEFIT [11] and BENEFIT-EXT [13] and the two phase II studies in which 
Fig. 2 Mean ( \pm standard deviation) belatacept serum concentration-time profiles after multiple doses of $5 \mathrm{mg} / \mathrm{kg}$ ( $n=14$; study IM103100) or $10 \mathrm{mg} / \mathrm{kg}(n=10$; study IM103047) in kidney transplant recipients at steady state. Time time post previous dose

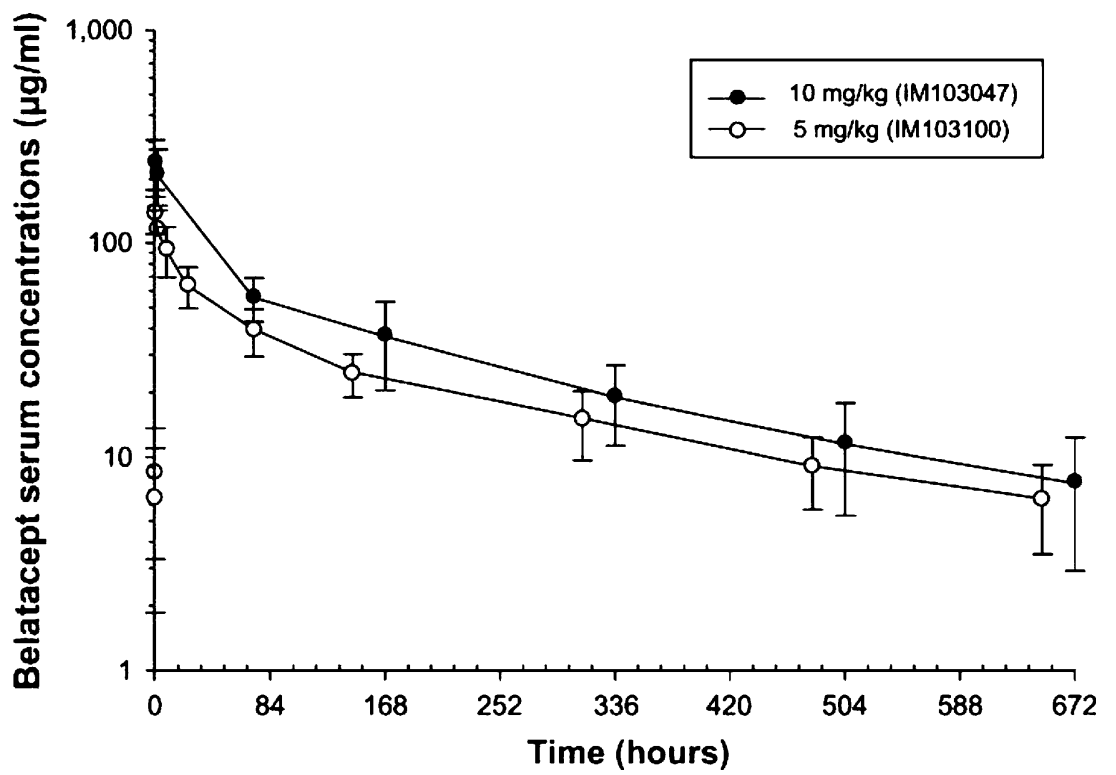

belatacept pharmacokinetics were estimated, belatacept doses of $10 \mathrm{mg} / \mathrm{kg}$ followed by $5 \mathrm{mg} / \mathrm{kg}$ were administered as part of an LI or MI regimen, with the same concomitant immunosuppressive treatment of basiliximab induction and mycophenolate mofetil (MMF) and corticosteroid taper (see Fig. 1). Serum concentration-time profiles of belatacept following multiple doses of 5 or $10 \mathrm{mg} / \mathrm{kg}$ are shown in Fig. 2; steady-state pharmacokinetic parameters of belatacept are summarized in Table 1. Exposure to belatacept increased in an approximately dose-proportional manner between 5 and $10 \mathrm{mg} / \mathrm{kg}$, suggesting linear pharmacokinetics in KTRs over this dose range. The variability of exposure to belatacept was relatively low, with the CV\% for $C_{\max }$ being 20 and $27 \%$ for the 5 and $10 \mathrm{mg} / \mathrm{kg}$ doses, respectively, and the $\mathrm{CV} \%$ for the $\mathrm{AUC}_{\tau}$ being 27 and $35 \%$, respectively. Consistent with the approximate doseproportional increase of exposure to belatacept and linear pharmacokinetic, the CL and $t_{1 / 2}$ of belatacept were similar between the 5 and $10 \mathrm{mg} / \mathrm{kg}$ doses. The $V_{\text {ss }}$ of belatacept was low and approximately equal to the vascular volume, which is consistent with the physical property of belatacept as a large therapeutic protein, with distribution limited to the extracellular space.

Consistent with the doses and dosing schedules of the LI and MI regimens, belatacept $C_{\text {trough }}$ levels were similar between the LI and MI regimens on day 5 in the phase III studies BENEFIT and BENEFIT-EXT [11, 13] (Table 2). However, the MI regimen delivered twice the belatacept dose as the LI regimen between months 2 and 6 posttransplant, corresponding to the approximately two- to three-fold higher belatacept $C_{\text {trough }}$ for the MI regimen than for the LI regimen between months 2 and 7. Belatacept $C_{\text {trough }}$ was also similar between the LI and MI regimens from month 7 post-transplant because both regimens had
Table 1 Belatacept pharmacokinetic parameters at steady state following multiple intravenous doses of 5 and $10 \mathrm{mg} / \mathrm{kg}$

\begin{tabular}{lll}
\hline $\begin{array}{l}\text { Pharmacokinetic } \\
\text { parameter }\end{array}$ & $\begin{array}{l}5 \mathrm{mg} / \mathrm{kg} \\
(N=14)\end{array}$ & $\begin{array}{l}10 \mathrm{mg} / \mathrm{kg} \\
(N=10)\end{array}$ \\
\hline$C_{\max }(\mu \mathrm{g} / \mathrm{mL})$ & $136(20)$ & $238(27)$ \\
$\mathrm{AUC}_{\tau}(\mu \mathrm{g} \cdot \mathrm{h} / \mathrm{mL})$ & $13,587(27)$ & $21,241(35)$ \\
$t_{1 / 2}($ days $)$ & $8.0(3.1-11.9)^{\mathrm{a}}$ & $8.5(6.1-15.1)$ \\
$\mathrm{CL}(\mathrm{mL} / \mathrm{h} / \mathrm{kg})$ & $0.49(27)^{\mathrm{b}}$ & $0.47(27)$ \\
$V_{\mathrm{ss}}(\mathrm{L} / \mathrm{kg})$ & $0.12(20)^{\mathrm{b}}$ & $0.11(30)$ \\
\hline
\end{tabular}

Values are expressed as geometric mean (CV\%) unless specified otherwise

$A U C_{\tau}$ area under the concentration-time curve for one dosing interval (4 weeks), $C L$ total body clearance, $C_{\max }$ peak serum concentration, $t_{1 / 2}$ elimination half-life, $V_{s S}$ volume of distribution at steady state, $\mathrm{CV} \%$ coefficient of variation percentage

${ }^{\text {a }}$ Median (range)

b $N=12$ for these parameters

the same dose and dosing schedule of $5 \mathrm{mg} / \mathrm{kg}$ every 4 weeks during the maintenance phase.

\subsection{Pharmacodynamics of Belatacept in KTRs}

The ideal assessment of pharmacodynamic activity of belatacept in KTRs would involve directly measuring the inhibition of the recipient's alloresponse to the donor antigens; however, direct measurement of the inhibition of alloresponses by belatacept in a clinical setting poses numerous technical challenges, including requirements for donor tissue, complex sample processing, complex cellular assays, and potential drug washout diminishing the immunosuppressive effect to be measured. Previous in vitro studies using mixed lymphocyte reaction assays 
Table 2 Belatacept serum trough concentration in the BENEFIT [11] and BENEFIT-EXT [13] studies

\begin{tabular}{|c|c|c|c|c|c|c|c|c|c|c|}
\hline \multirow[t]{3}{*}{ Studies/Regimens } & \multicolumn{10}{|l|}{ Time } \\
\hline & \multirow{2}{*}{$\begin{array}{l}\text { Day } \\
5\end{array}$} & \multicolumn{9}{|l|}{ Weeks } \\
\hline & & 8 & 12 & 16 & 24 & 36 & 52 & 104 & 128 & 156 \\
\hline \multicolumn{11}{|l|}{ BENEFIT } \\
\hline LI regimen $(n)$ & 208 & 197 & 183 & 176 & 177 & 179 & 173 & 166 & 164 & 102 \\
\hline$C_{\text {trough }}{ }^{\mathrm{a}}(\mu \mathrm{g} / \mathrm{mL})$ & $34.8(59)$ & $8.4(49)$ & $7.0(54)$ & $6.8(53)$ & $3.5(59)$ & $3.5(81)$ & $3.4(63)$ & $4.1(55)$ & $5.0(54)$ & $4.7(62)$ \\
\hline MI regimen $(n)$ & 202 & 194 & 190 & 174 & 171 & 171 & 162 & 152 & 150 & 97 \\
\hline$C_{\text {trough }}{ }^{\mathrm{a}}(\mu \mathrm{g} / \mathrm{mL})$ & $35.4(32)$ & $23.7(59)$ & $26.2(38)$ & $10.7(79)$ & $7.8(66)$ & $3.8(60)$ & $3.8(51)$ & $4.5(58)$ & $5.3(65)$ & $5.7(57)$ \\
\hline \multicolumn{11}{|l|}{ BENEFIT-EXT } \\
\hline LI regimen $(n)$ & 150 & 146 & 139 & 130 & 131 & 126 & 114 & 107 & 104 & 95 \\
\hline$C_{\text {trough }}{ }^{\mathrm{a}}(\mu \mathrm{g} / \mathrm{mL})$ & $35.7(31)$ & $9.6(60)$ & $8.5(83)$ & $8.0(57)$ & $4.3(57)$ & $3.9(52)$ & $4.3(57)$ & $4.1(71)$ & $5.6(66)$ & $5.3(60)$ \\
\hline MI regimen $(n)$ & 155 & 151 & 141 & 128 & 136 & 130 & 119 & 112 & 101 & 89 \\
\hline$C_{\text {trough }}{ }^{\mathrm{a}}(\mu \mathrm{g} / \mathrm{mL})$ & $38.3(54)$ & $26.4(40)$ & $27.7(64)$ & $12.9(46)$ & $9.3(46)$ & $4.2(61)$ & $4.1(54)$ & $4.8(102)$ & $5.6(63)$ & $6.0(86)$ \\
\hline
\end{tabular}

$C_{\text {trough }}$ serum trough concentration, $C V \%$ percentage coefficient of variation, $L I$ less intensive, $M I$ more intensive

${ }^{a}$ Values are expressed as geometric mean $(\mathrm{CV} \%)$

with purified human dendritic cells and T cells demonstrated that the blockade of CD80/CD86-mediated costimulation by belatacept and thus the inhibition of $\mathrm{T}$ cell activation and proliferation during an alloimmune response correlated with CD86 receptor occupancy [6]. These results suggest that CD86 receptor occupancy could be a surrogate measure of the immunosuppressive pharmacodynamic effect of belatacept in clinical studies in KTRs. Thus, the pharmacodynamics of belatacept in KTRs in vivo was assessed via its binding to CD86 receptors on monocytes in a corticosteroid-avoidance phase II study (IM103034) in which belatacept was administered in two study groups (belatacept + MMF and belatacept + sirolimus) [9].

Approximately $90 \%$ CD86 receptor occupancy of belatacept was needed for maximal inhibition of lymphocyte proliferation and cytokine interferon- $\gamma$ production in vitro [6]. With the administration of the belatacept MI regimen, high CD86 receptor occupancy (94\%) was observed during the initial phase post-transplant as free CD86 receptors decreased to a minimum (Fig. 3a), suggesting maximal blockade of CD80/CD86-mediated co-stimulations by belatacept to inhibit $\mathrm{T}$ cell activation and proliferation during this critically important initial phase to prevent acute rejection as the transplanted organ underwent engraftment. A gradual increase of free CD86 receptors was observed through the maintenance phase as the belatacept $C_{\text {trough }}$ decreased. The levels of free CD86 receptors observed over time translated into mean CD86 receptor occupancy by belatacept of $94,88,86,75,67$, and $65 \%$ at post-transplant days $5,14,28,84,168$, and 364 , respectively, suggesting a gradually reduced pharmacodynamic effect. The slow decrease in CD86 receptor occupancy corresponded to a slow decrease in belatacept $C_{\text {trough }}$. In the two belatacept groups (belatacept + MMF and belatacept + sirolimus), the geometric mean $C_{\text {trough }}$ of belatacept ranged from $34.9-39.5 \mu \mathrm{g} / \mathrm{mL}$ on post-transplant day 5 . The geometric mean $\mathrm{C}_{\text {trough }}$ of belatacept decreased to $29.4-34.4 \mu \mathrm{g} / \mathrm{mL}$ at day $14,23.5-32.5 \mu \mathrm{g} / \mathrm{mL}$ at day $28,27.6-30.5 \mu \mathrm{g} / \mathrm{mL}$ at day 84 , and $8.0-10.1 \mu \mathrm{g} / \mathrm{mL}$ at day 168 , and it further decreased to $\sim 4 \mu \mathrm{g} / \mathrm{mL}$ at day 364 of the maintenance phase in this study. To determine if exposure to belatacept in KTRs in vivo down-regulated the expression of CD86 receptors, the expression of total CD86 receptors on monocytes was also assessed. Following a transient (albeit insignificant) decrease in total CD86 expression on day 5, no significant change in total expression of CD86 receptors was observed after day 5 post-transplant compared with baseline pre-transplant (data not shown).

The pharmacokinetic and pharmacodynamic relationship between free CD86 receptors and belatacept concentration in KTRs in vivo were analyzed using an inhibitory $E_{\max }$ model. The baseline free CD86 receptor level $E_{0}$ and maximal decrease in free CD86 receptors in the presence of belatacept were estimated to be 9,820 (95\% CI 8,319-11,320) and 9,144 (95 \% CI 7,662-10,619) MESF, respectively, in KTRs. The effective concentrations of belatacept required to decrease free $\mathrm{CD} 86$ receptors by $50 \%\left(E C_{50}\right)$ and $90 \%\left(E C_{90}\right)$ were estimated to be 2.4 (95\% CI 1.2-3.5) and 21.2 (95\% CI 10.7-31.7) $\mu \mathrm{g} / \mathrm{mL}$, respectively. The maximal CD86 receptor occupancy by belatacept was estimated to be $93 \%$ (95\% CI 88-98) using this model, which was in good agreement with observed value of $94 \%$ during the initial phase posttransplant. The model-predicted free CD86 receptor expression levels versus belatacept concentrations were in good agreement with observed values in a visual predicative check (Fig. 3b). 

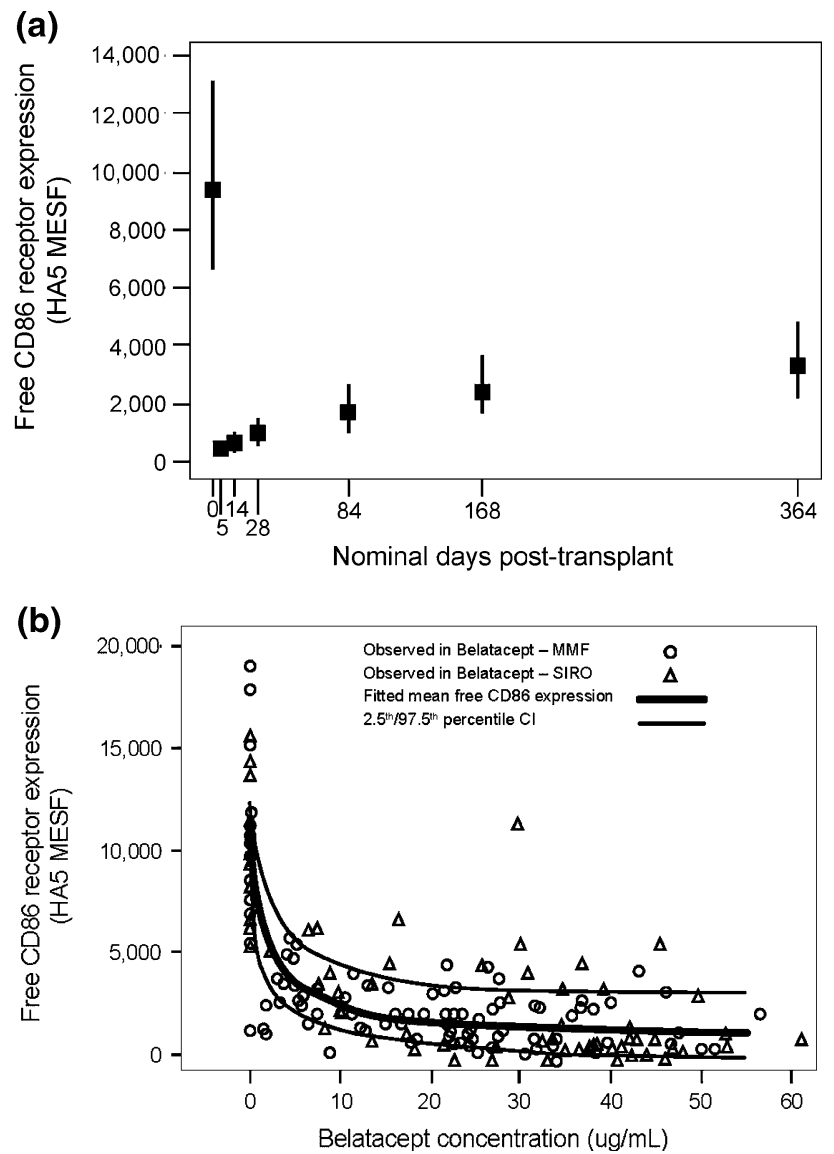

Fig. 3 Free CD86 receptor expression on monocytes following belatacept treatment in KTRs. a Plot of free CD86 receptor level versus nominal visit time for KTRs treated with the belatacept MI regimen in a phase II corticosteroid-avoidance study. Solid symbols indicate fitted mean of free CD86 receptor level at each timepoint. Lines indicate $95 \%$ confidence intervals. b Visual predictive check of free CD86 receptor expression levels versus belatacept concentrations on whole peripheral blood monocytes in KTRs treated with the belatacept MI regimen in a phase II corticosteroid-avoidance study. Observed and model-predicted median, 2.5th, and 97.5th percentiles are presented. Open circles represent observed datapoints from KTRs treated with belatacept + MMF. Open triangle represent observed datapoints from KTRs treated with belatacept + SIRO. $C I$ confidence interval, KTR kidney transplant recipient, $L I$ less intensive, MESF molecules of equivalent soluble fluorochrome, $M I$ more intensive, $M M F$ mycophenolate mofetil, SIRO sirolimus

\subsection{Immunogenicity Assessment of Belatacept in KTRs}

In the phase II dose-ranging study IM103100 and its LTE $[14,18]$, and in the phase III studies BENEFIT [11] and BENEFIT-EXT [13] through at least 3 years of exposure to belatacept, 847 belatacept-treated KTRs had $\geq 1$ posttreatment immunogenicity assessments performed. KTRs treated with belatacept received the same concomitant immunosuppressive medications of basiliximab induction and MMF and corticosteroid taper in these three studies.

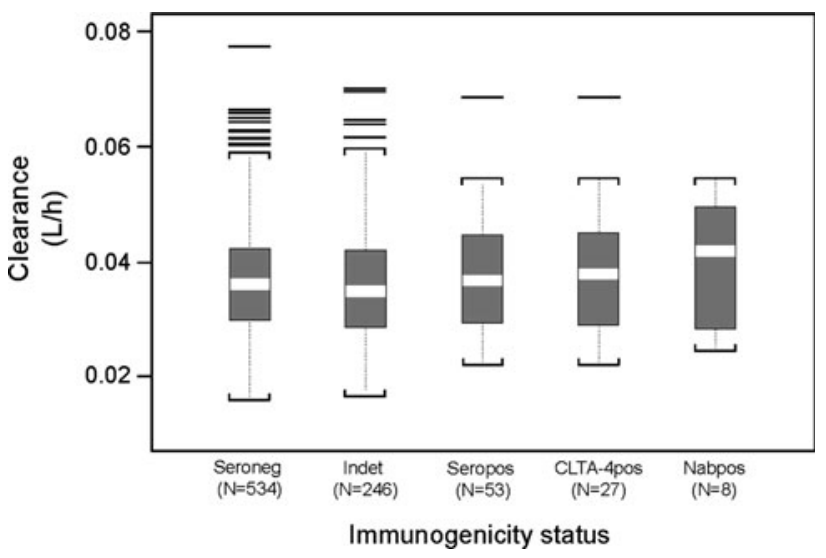

Fig. 4 Box and whisker plots of Bayesian-predicted clearance of belatacept in KTRs who were seronegative, indeterminate, seropositive, and seropositive against the modified CTLA-4 region or neutralizing antibodies-positive with anti-belatacept antibodies in the BENEFIT, BENEFIT-EXT, and phase II long-term extension studies. The horizontal line within each box represents the median. The lower and upper ends of the boxes represent the 25th and 75th percentiles, and the whiskers represent the 5th and 95th percentiles. The horizontal lines outside the whiskers represent clearance values that were outside the 95th percentile. CTLA-4 cytotoxic T-lymphocyteassociated antigen 4, CTLA-4pos cytotoxic T-lymphocyte-associated antigen 4-positive, Indet indeterminate, KTR kidney transplant recipient, $N A b$ neutralizing antibodies, NAbpos neutralizing antibodies-positive, Seroneg seronegative, Seropos seropositive

Of 847 KTRs, 45 (5.3\%) were found to be seropositive for anti-belatacept antibodies at some point during treatment. Of these 45 KTRs, 20 also tested seropositive prior to the administration of belatacept. Ten of 153 KTRs $(6.5 \%)$ were seropositive after discontinuation of treatment with belatacept. Similar proportions of patients had either transient or persistent antibodies (2.4 and $2.5 \%$, respectively).

Anti-belatacept antibody titers in seropositive KTRs were generally low (all $\leq 640$ ) and were not consistently directed towards any particular part of the belatacept molecule. Of 45 KTRs who had anti-belatacept antibodies, 29 had anti-belatacept antibodies specific to the modified CTLA4 portion of belatacept. Of these 29 , eight were positive for neutralizing antibodies, 19 were designated indeterminate for neutralizing antibodies, and two were negative for neutralizing antibodies. Antibody titers had either decreased or were no longer detectable at a subsequent timepoint for the majority of KTRs who tested positive at any timepoint. Continued dosing with belatacept did not lead to an increase in anti-belatacept titers. Integrated analysis using data from the phase II study IM103100 and its LTE and from the BENEFIT and BENEFIT-EXT studies indicated no apparent effect of anti-belatacept antibodies on belatacept clearance in KTRs (Fig. 4). 


\section{Discussion}

The pharmacokinetics and pharmacodynamics of a drug in its target patient population is an important and integral part of drug development that provides important information to guide its clinical use. During the clinical development of belatacept in KTRs, two dosing regimens (LI and MI) were developed and tested in the phase II study IM103100 [18] and the phase III studies BENEFIT [11] and BENEFIT-EXT [13]. The development and selection of belatacept LI and MI regimens for testing in phase II and III studies were guided by integrated non-clinical pharmacology, clinical pharmacokinetics, pharmacodynamics, safety, and efficacy data from multiple phase I and II studies [19]. The belatacept LI regimen was subsequently approved for the prophylaxis of organ rejection in adult KTRs based on results from the phase III studies BENEFIT and BENEFIT-EXT, as well as its overall benefit/risk profile versus ciclosporin [11,13]. This is the first report of the pharmacokinetics, pharmacodynamics, and immunogenicity data of belatacept in KTRs. Here we show that belatacept exposure increases in an approximately doseproportional manner between doses of 5 and $10 \mathrm{mg} / \mathrm{kg}$, which are the clinical doses approved in KTRs. Belatacept also exhibits relatively low pharmacokinetic variability with predictable exposure upon intravenous dosing in KTRs. Furthermore, two phase III studies with a constant maintenance regimen $(5 \mathrm{mg} / \mathrm{kg}$ every 4 weeks) in KTRs demonstrated that the $C_{\text {trough }}$ of belatacept is consistently maintained up to 3 years post-transplant, which is consistent with clinical practice in phase II and III studies that TDM of belatacept was not required.

The pharmacokinetics of belatacept provided clinical guidance for the approved LI regimen in KTRs. Consistent with belatacept being a large therapeutic protein, the $V_{\mathrm{ss}}$ of belatacept suggests that its distribution is limited to the extracellular space. The belatacept MI dosing regimen delivers twice the total belatacept dose as the approved LI regimen during months 2-6 post-transplant. Accordingly, the $C_{\text {trough }}$ of belatacept following administration of the MI regimen was approximately two to three times greater than the $C_{\text {trough }}$ of belatacept following administration of the LI regimen during months 2-7 post-transplant. While the exposure to belatacept in KTRs was the same between the LI and MI regimens during month 1 and after month 7 post-transplant, clinical efficacy and safety data suggest that the increased exposure from the MI regimen during months 2-7 post-transplant resulted in a slightly less favorable safety profile, with no additional efficacy benefit $[11,13,20]$, supporting the LI regimen as the recommended regimen in KTRs.

Understanding the pharmacokinetics and pharmacodynamics of a drug in its target patient population provides important information regarding its mechanism of action in vivo and informs the exposure that may be required to achieve the desired level of activity. Here we show that belatacept demonstrates predictable concentration-dependent pharmacodynamics in KTRs, as measured by its binding to CD86 receptors, which is consistent with its intended mechanism of action. In vitro studies have shown that CD86 receptor saturation by belatacept correlates with inhibition of T cell alloresponses [6]. A CD86 receptor competition assay performed on blood samples from belatacept-treated patients in the phase II corticosteroidavoidance study evaluated the extent to which belatacept occupied its target receptor on the surface of APCs in peripheral blood [9]. The sampling schedule allowed for the evaluation of CD86 receptor occupancy on day 5 following initial intravenous infusion of belatacept and evaluation of the change in occupancy over time as the dosing interval and concentrations varied. All blood sampling occurred prior to dosing, and thus the measured CD86 occupancies reported here represent assessment at $C_{\text {trough }}$ and are the minimal receptor occupancies achieved. For approximately the first week following each infusion, receptor occupancy was expected to be high ( $>90 \%$ of the maximum value). The high level of CD86 occupancy in the peri-transplant period is necessary for full blockade of $\mathrm{T}$ cell co-stimulation and is critical for the prevention of graft rejection at the time when risk of acute rejection is greatest. As the concentration of belatacept decreased during the period between the initial and maintenance phases, free CD86 receptor levels increased, reflecting a decrease in receptor occupancy. Less immunosuppression with belatacept is required during the intermediate and maintenance phases, since the risk of graft rejection decreases over time. The pharmacokinetic and pharmacodynamic relationship of CD86 receptor occupancy by belatacept in KTRs was further assessed quantitatively using a non-linear mixed effect inhibitory $E_{\max }$ model. The model parameter estimates indicated that belatacept $C_{\text {trough }}$ during the critically important peri-transplant period was greater than that needed for $90 \%$ CD86 receptor occupancy, suggesting maximal pharmacodynamic activity was achieved to prevent acute rejection during this period [6].

Zhou et al. [17] previously characterized the population pharmacokinetics and exposure-response of belatacept in patients with KTR. They reported that the only significant covariates affecting CL and volume at distribution was baseline body weight. Other covariates were also tested for their potential impact on the pharmacokinetics of belatacept, but none were deemed significant, including age, sex, race, renal function, hepatic function, presence of diabetes, and concomitant dialysis. They found that belatacept exposure and dosing regimen were not predictive of acute rejection. However, higher exposures of belatacept 
associated with the MI dosing regimen were associated with an increased risk of serious adverse events, including serious infections and central nervous system events [17].

This is the first report of the immunogenicity of belatacept in KTRs. The development of anti-drug antibodies is common following administration of therapeutic proteins. Anti-drug antibodies may reduce the efficacy of a drug by lowering the molecule's $t_{1 / 2}$ in the circulatory system or preclude repeat dosing if re-administration is to cause a strong immune reaction $[2,21]$. Here we report a low incidence rate for the formation of anti-belatacept antibodies in KTRs. This low incidence rate could be due to the inhibition of antibody formation by belatacept, a possible effect based on its known mechanism of action, in addition to the effect of concomitant immunosuppressive agents, which may also inhibit formation of anti-belatacept antibodies. In KTRs who developed anti-belatacept antibodies, the detection of these antibodies appeared to be transient, and their titers were generally low over the course of treatment. More importantly, anti-belatacept antibodies had no apparent effect on the exposure to belatacept. However, given the low incidence of immunogenicity with the approved LI regimen (2\%), definitive conclusions regarding the impact of anti-belatacept antibody formation on safety or efficacy could not be made.

No formal drug-drug interaction study has been completed with belatacept except with mycophenolic acid (MPA), which is frequently co-administered with belatacept as a background immunosuppressant. Patients who received belatacept had a mean dose-normalized MPA $C_{\max }$ and $\mathrm{AUC}_{\tau}$ of 20 and $40 \%$ higher, respectively, than patients who received ciclosporin [4]. However, this effect was likely due to ciclosporin, which is known to inhibit enterohepatic recirculation and require adjustment of the MPA dose when converting to or off ciclosporin [22]. Several small-molecule drugs used in KTRs are metabolized or inhibitors of cytochrome P450 (CYP) 3A4 and P-glycoprotein and thus can have multiple drug-drug interactions [22]. In contrast, belatacept is a large-molecule therapeutic protein not expected to be affected by other drugs [23]. However, other biologics have been shown to cause interaction indirectly by down-regulation of CYP enzymes through cytokine release [24], which have been shown to be blocked by belatacept in vitro but not in vivo [4].

\section{Conclusion}

This is the first report summarizing the pharmacokinetics, pharmacodynamics, and immunogenicity results of belatacept in KTRs. Belatacept exhibited linear pharmacokinetics and an approximately dose-proportional increase in exposure in KTRs. CD86 receptor occupancy data also suggested concentration-dependent pharmacodynamics of belatacept in KTRs. The incidence of developing anti-belatacept antibodies in KTRs was low and had no impact on belatacept exposure. These data, along with confirmation from phase III studies, provides justification for the belatacept LI dosing regimen in KTRs.

Acknowledgments This work was supported by Bristol-Myers Squibb Company.

The authors would like to express their gratitude to investigators and patients who participated in the clinical studies, and to Zoe Tzogas for her contribution to the immunogenicity monitoring. We also would like to thank Anju Anne Roy, PhD, for assistance in preparing the manuscript and CodonMedical (a division of KnowledgePoint360 Group) for providing editorial support.

Financial disclosures Janice Pursley, Eric Masson, Nadia McGirr, Elizabeth Proszynski, Yun Shen, Kathleen Soucek, Robert Townsend, Xiaoli You, and Zexun Zhou are employees of Bristol-Myers Squibb Company; Eric Masson is a stockholder of Bristol-Myers Squibb Company; Dong Geng, Jinshan Shen, Dianna Wu, and Ping Zhan are former employees of Bristol-Myers Squibb Company.

Open Access This article is distributed under the terms of the Creative Commons Attribution Noncommercial License which permits any noncommercial use, distribution, and reproduction in any medium, provided the original author(s) and the source are credited.

\section{References}

1. Meier-Kriesche HU, Schold JD, Srinivas TR, et al. Lack of improvement in renal allograft survival despite a marked decrease in acute rejection rates over the most recent era. Am J Transplant. 2004;4(3):378-83.

2. Halloran PF. Immunosuppressive drugs for kidney transplantation. N Engl J Med. 2004;351(26):2715-29.

3. Schiff J, Cole E, Cantarovich M. Therapeutic monitoring of calcineurin inhibitors for the nephrologist. Clin J Am Soc Nephrol. 2007;2(2):374-84.

4. Nujolix ${ }^{\circledR}$ (belatacept) [package insert]. Princeton: Bristol-Myers Squibb Co.; 2013.

5. Larsen CP, Pearson TC, Adams AB, et al. Rational development of LEA29Y (belatacept), a high-affinity variant of CTLA4-Ig with potent immunosuppressive properties. Am J Transplant. 2005;5(3):443-53.

6. Latek R, Fleener C, Lamian V, et al. Assessment of belataceptmediated costimulation blockade through evaluation of CD80/86receptor saturation. Transplantation. 2009;87(6):926-33.

7. Paweletz C, Andersen JN, Pollock R, et al. Identification of direct target engagement biomarkers for kinase-targeted therapeutics. PLoS One. 2011;6(10):e26459.

8. Valencia X. An open-label pharmacokinetic study in de novo renal transplant subjects receiving a belatacept-based immunosuppressant regimen. Princeton: Bristol-Myers Squibb; 2009. (Data on file).

9. Ferguson R, Grinyo J, Vincenti F, et al. Immunosuppression with belatacept-based, corticosteroid-avoiding regimens in de novo kidney transplant recipients. Am J Transplant. 2011;11(1):66-76.

10. Johnson A. Open-label, randomized, controlled, multiple-dose study of efficacy and safety of BMS-224818 as part of a 
quadruple drug regimen in renal transplant recipients (pharmacokinetic sub-study). Princeton: Bristol-Myers Squibb; 2008. (Data on file).

11. Vincenti F, Charpentier B, Vanrenterghem Y, et al. A phase III study of belatacept-based immunosuppression regimens versus cyclosporine in renal transplant recipients (BENEFIT study). Am J Transplant. 2010;10(3):535-46.

12. Larsen C, Grinyo J, Medina-Pestana J, et al. Belatacept-based regimens vs a cyclosporine-based regimen in kidney transplant recipients: 2-year results from the BENEFIT and BENEFIT-EXT studies. Transplantation. 2010;90(12):1528-35.

13. Durrbach A, Pestana JM, Pearson T, et al. A phase III study of belatacept versus cyclosporine in kidney transplants from extended criteria donors (BENEFIT-EXT study). Am J Transplant. 2010;10(3):547-57.

14. Vincenti F, Blancho G, Durrbach A, et al. Five-year safety and efficacy of belatacept in renal transplantation. J Am Soc Nephrol. 2010;21(9):1587-96.

15. Neoral ${ }^{\circledR}$ Soft Gelatin Capsules (cyclosporine capsules, USP) MODIFIED; Neoral ${ }^{\circledR}$ Oral Solution (cyclosporine oral solution, USP) MODIFIED [package insert]. Basel: Novartis International AG; 2012.

16. Myler H, Phillips KR, Dong H, et al. Validation and life-cycle management of a quantitative ligand-binding assay for the measurement of Nulojix ${ }^{\circledR}$, a CTLA-4-Fc fusion protein, in renal and liver transplant patients. Bioanalysis. 2012;4(10):1215-26.
17. Zhou Z, Shen J, Hong Y, et al. Time-varying belatacept exposure and its relationship to efficacy/safety responses in kidney-transplant recipients. Clin Pharmacol Ther. 2012;92(2):251-7.

18. Vincenti F, Larsen C, Durrbach A, et al. Costimulation blockade with belatacept in renal transplantation. N Engl J Med. 2005; 353(8):770-81.

19. Shen J, Townsend R, Kaul S. An integrated approach to develop belatacept dosing regimens for phase 3 studies in renal transplant subjects [abstract no. OII-A-4]. Clin Pharmacol Ther. 2011; 89(Suppl 1):S37.

20. Grinyo J, Charpentier B, Pestana JM, et al. An integrated safety profile analysis of belatacept in kidney transplant recipients. Transplantation. 2010;90(12):1521-7.

21. Wadhwa M, Thorpe R. Unwanted immunogenicity: lessons learned and future challenges. Bioanalysis. 2010;2(6):1073-84.

22. Kuypers DR. Immunotherapy in elderly transplant recipients: a guide to clinically significant drug interactions. Drugs Aging. 2009;26(9):715-37.

23. Seitz K, Zhou H. Pharmacokinetic drug-drug interaction potentials for therapeutic monoclonal antibodies: reality check. J Clin Pharmacol. 2007;47:1104-18.

24. Lee J-I, et al. CYP-mediated therapeutic protein-drug interactions. Clin Pharmacokinet. 2010;49(5):295-310. 